La

Révolution

française

\section{La Révolution française}

Cahiers de l'Institut d'histoire de la Révolution française

$8 \mid 2015$

Entre la Révolution et l'Empire : une nouvelle politique dans l'océan Indien

\title{
La médiation des dubashes
}

Un aspect de la politique française en Inde dans la seconde moitié du XVIII ${ }^{\mathrm{e}}$ siècle

Julie Marquet

\section{OpenEdition}

\section{Journals}

Édition électronique

URL : http://journals.openedition.org/lrf/1259

DOI : $10.4000 /$ Irf. 1259

ISSN : 2105-2557

Éditeur

IHMC - Institut d'histoire moderne et contemporaine (UMR 8066)

Édition imprimée

Date de publication : 23 juin 2015

Référence électronique

Julie Marquet, «La médiation des dubashes », La Révolution française [En ligne], 8 | 2015, mis en ligne le 24 juin 2015, consulté le 14 février 2020. URL : http://journals.openedition.org//rf/1259 ; DOI

$10.4000 /$ Irf.1259

Ce document a été généré automatiquement le 14 février 2020.

(C) La Révolution française 


\title{
La médiation des dubashes
}

\author{
Un aspect de la politique française en Inde dans la seconde moitié du \\ XVIII ${ }^{\mathrm{e}}$ siècle
}

Julie Marquet

1 Le jour de la nouvelle année, les marchands et les notables indiens de Pondichéry viennent présenter leurs respects et offrir des présents au gouverneur. A cette occasion, le courtier de ce dernier établit l'ordre hiérarchique strict selon lequel chacun doit se présenter. Le $1^{\mathrm{er}}$ janvier 1765 , Sami Pillai, le dubash (interprète) du tribunal de la Chaudrie, rapporte que Candapa, le courtier du gouverneur, n'a pas respecté «ce qui avait été en pratique depuis longtemps », et l'a placé après le nayinar (grand prévôt) ${ }^{1}$.

2 La médiation du courtier est nécessaire au bon déroulement de la cérémonie. Il introduit et présente les notables et les principaux agents indiens du comptoir. En même temps, il prend en charge une partie du protocole, en attribuant à chacun une place précise dont dépendra la considération du gouverneur - et il agit de manière à réduire le prestige de Sami Pillai, un proche de son grand rival Tiruvengadam III. L'acte de médiation a ici une double dimension : il y a mise en relation et intervention dans la relation. L'intermédiaire est à la fois un agent qui facilite les transactions, et un acteur de ces transactions.

3 La place des intermédiaires dans les processus d'interaction et leur implication dans les processus qu'ils médiatisent a d'abord été étudiée par les sociologues et les anthropologues ${ }^{2}$. Dans son étude du kijaji javanais, Clifford Geertz a souligné la complexité du positionnement de l'intermédiaire culturel ${ }^{3}$. Ce positionnement, entre ancrage local et représentation nationale, évolue à Java avec le développement du système d'éducation et des partis politiques. L'intermédiaire culturel compose en fonction d'une part des possibilités et d'autre part de ses différents niveaux d'appartenance. Les historiens se sont récemment intéressés au rôle des intermédiaires et aux modalités de la mise en contact, dans une perspective d'histoire impériale - en étudiant les relais indigènes de l'expansion impériale et les acteurs des sociétés coloniales $^{4}$, ou dans une perspective d'histoire globale - en éclairant les connexions et les circulations des hommes, des biens, ou des savoirs ${ }^{5}$. Cet intérêt nouveau de 
l'historiographie a été marqué par la publication de l'ouvrage collectif The Brokered World: Go-Betweens and Global Intelligence, codirigé par Simon Schaffer, Lissa Roberts, Kapil Raj et James Delbourgo. Les articles de The Brokered World proposent une réflexion sur les manières de (se) définir (de) l'intermédiaire, sur les processus de (co)production des savoirs dans lesquels il se trouve engagé, et sur les formes de liens tissés avec les compagnies européennes ou les administrateurs coloniaux, entre les années 1770 et $1820^{6}$. Plusieurs articles portent sur l'Inde, et ouvrent des perspectives pour l'histoire des établissements français. Les travaux sont rares sur l'implantation française en Inde, et concernent peu les processus d'interaction?

4 Cet article s'intéresse aux interactions entre le gouvernement français et ses intermédiaires indiens, à Pondichéry, dans la seconde moitié du XVIII ${ }^{\mathrm{e}}$ siècle. Le rôle de ces intermédiaires dans l'administration coloniale évolue au cours de cette période. Le gouvernement, en tant que forme politique, est fortement lié aux affaires privées, aux entreprises commerciales, et aux jeux de pouvoir personnels. En tant qu'organe, il est composé du gouverneur et d'un petit noyau d'administrateurs, et tend à se confondre avec l'administration coloniale. Celle-ci est très réduite dans la seconde moitié du XVIII ${ }^{e}$ siècle, en raison de la déprise progressive de la France en Inde : Pondichéry est prise et occupée trois fois par les Britanniques entre 1761 et 1763 (guerre de Sept Ans), entre 1778 et 1784 (guerre d'indépendance américaine), et entre 1793 et 1816 (Révolution française et guerres napoléoniennes) ${ }^{8}$. En dehors des périodes d'occupation et de vacance du gouvernement français, l'administration coloniale assume les prérogatives de l'autorité politique supérieure (police, justice, relations diplomatiques...). L'exercice de la souveraineté et du gouvernement des populations détermine la nature de l'établissement colonial, mais elle ne correspond pas à une emprise cohérente et fermement établie. Les Français n'ont ni les moyens, ni la volonté d'assurer un contrôle strict des circuits commerciaux et des populations locales. Au contraire, ils s'insèrent dans des réseaux et des structures économiques ou sociales préexistants, et s'appuient sur des relais locaux.

5 L'enjeu est ici de centrer la réflexion sur un groupe particulier d'intermédiaires indiens engagés dans l'administration coloniale, pour essayer de comprendre comment leur position et leur statut sont (re)définis par le jeu des intérêts réciproques. L'articulation des rapports d'intérêt, d'utilité, voire de dépendance entre les dubashes et l'administration française repose sur une dynamique de rapprochement et de mise à l'écart, voire, ainsi que l'ont analysé Jane Burbank et Frederick Cooper dans leur étude des empires, une dynamique d' " intégration » et de " différenciation" ${ }^{9}$ ".

6 Le dubash, est, littéralement, un «homme des deux langues ${ }^{10} »$. Cet intermédiaire est donc d'abord défini par sa maitrise des langues, nécessaire aux Français pour communiquer avec les populations locales. Mais il est plus qu'un interprète: dans la documentation, les termes « dubash » et « courtier » sont utilisés indifféremment, pour désigner un individu particulier ou poste global de médiation. A Pondichéry, les dubashes ont des fonctions multiples et souvent indissociées. Employés par des particuliers, ils sont à la fois traducteurs, entremetteurs, majordomes en charge de l'intendance et de la domesticité, prêteurs, courtiers ou partenaires commerciaux. Ils peuvent entrer au service de la Compagnie française des Indes ou de l'administration $\mathrm{du}$ comptoir, comme tabellions ou huissiers du tribunal local, agents de police, interprètes ou émissaires. Le courtier en chef - parfois secondé par un courtier adjoint - occupe la place principale. Ces différentes fonctions sont fortement hiérarchisées et 
revêtent un caractère plus ou moins honorifique. Dès lors, passer de l'une à l'autre constitue un enjeu dans les carrières des dubashes.

7 Jusqu'à la suppression de la Compagnie française des Indes en 1769, le courtier en chef était le premier relais de la politique française à Pondichéry. Il concentrait l'ensemble des fonctions commerciales, diplomatiques, politiques et sociales, tout en poursuivant ses activités privées ${ }^{11}$. A partir de 1769 , ses fonctions sont disjointes et attribuées à différents agents: on distingue désormais un Diwan (conseiller politique et diplomatique), un «chef des Indiens", un interprète et un courtier pour les affaires commerciales $^{12}$.

8 Ces fonctions font l'objet de négociations et de revendications permanentes. Trois familles sont en concurrence pour les principaux postes de médiation. L'une d'entre elles, celle de Tiruvengadam, réussit à se maintenir au plus haut niveau de l'administration coloniale: Ananda Ranga Pillai (1709-1761) est courtier en chef au milieu du siècle ; son neveu Tiruvengadam III occupe de manière instable les principaux postes de 1769 à sa mort en 1791 ; le fils de ce dernier, Tiruvengadam IV, est chef des Indiens de 1791 à son décès en 1801.

9 Ces trois dubashes ont produit une importante documentation officielle (courriers et mémoires adressés à l'administration, pièces d'actions en justice) ou privée (documents comptables, journaux personnels). En dehors du journal d'Ananda Ranga Pillai, cette documentation a très peu été étudiée et reste en grande partie inédite. Elle présente un intérêt particulier : l'historienne Kanakalatha Mukund souligne le fait qu'il existe peu de sources produites par la société tamoule sur les situations de rencontre coloniale au XVIII ${ }^{e}$ siècle, et Sanjay Subrahmanyam estime que les journaux des courtiers constituent un genre presque inédit à cette période en Inde du sud ${ }^{13}$.

10 On a mis en regard les documents de la famille Pillai avec les documents produits par l'administration coloniale (arrêtés et décisions des gouverneurs, ou du conseil, courriers des administrateurs...), afin de comprendre comment ils se complètent, se répondent, ou se contredisent. On a cherché à lire ces documents tout à la fois en suivant et en allant à rebours de leurs intentions ${ }^{14}$. Par exemple, les mémoires produits par les dubashes disent la médiation réussie et l'insertion dans les rouages de l'administration : il faut en examiner les modalités et les enjeux; ils taisent les jeux de pouvoir dans lesquels sont pris leurs auteurs : il faut dégager ces dynamiques, en allant contre le sens donné par le document ; ils imaginent la continuité du projet colonial, qui n'a jamais existé : il faut éclairer la discontinuité.

11 A travers l'analyse de ce corpus, on perçoit l'instabilité des positions des dubashes, liée à la tension entre leur investissement dans le projet impérial français et la mise à l'écart de l'administration coloniale. Ils y répondent par des stratégies liées à l'écrit, visant à garantir ou à sécuriser leur statut. On envisage, enfin, la manière dont les Français mobilisent leurs agents pour la compréhension et la prise en charge des structures sociales locales : ils peuvent faire le choix de maintenir, de renforcer ou d'inventer ces structures en fonction de l'utilité qu'ils leur attribuent. 


\section{Participer de l'implantation française ? Entre investissement et mise à l'écart des dubashes}

\subsection{Déjouer les concurrences, jouer du lien avec les Français}

12 La concurrence est très forte pour l'obtention des principaux postes de médiation, notamment pour celui de courtier en chef. Ces postes garantissent en effet une position influente dans la société locale, et, surtout, ils s'avèrent très lucratifs. Le courtier en chef, en plus de son traitement, reçoit des cadeaux lors de la conclusion de contrats ou de traités, touche divers droits et commissions, bénéficie d'exemptions de taxes, et réalise des opérations lucratives grâce à sa maîtrise de la circulation de l'information.

Le patronage du gouverneur et les rapports interpersonnels avec les agents français sont essentiels à l'obtention de ces postes convoités. En effet, le gouverneur nomme au poste de courtier en chef un dubash qui a préalablement servi ses intérêts privés ${ }^{15}$. Les dubashes intègrent donc l'administration lorsqu'ils ont déjà établi des rapports commerciaux ou financiers avec des marchands ou des administrateurs français. Ces derniers peuvent être en concurrence entre eux, ce qui alimente un micro système de "partis» dans l'espace du comptoir. Le parcours de Tiruvengadam III témoigne de l'importance du patronage dans l'obtention des postes et du poids des jeux de "partis " dans les retournements de fortune. Il assiste son oncle Ananda Ranga Pillai jusqu'à la prise de Pondichéry en 1761, date à laquelle il s'installe au sud de la ville, dans le comptoir danois de Tranquebar, où il est en relation avec les différents agents français. A partir du retour du gouvernement à Pondichéry en 1765, il cherche à obtenir le poste de courtier en chef, mais le gouverneur Law de Lauriston appointe et soutient Candappa, son dubash privé, jusqu'à son rappel en 1776. Tiruvengadam III fait jouer les différents niveaux d'autorité et la rivalité entre partis : il cherche avec insistance la recommandation et le soutien de conseillers à Pondichéry et écrit en France, dès 1765 . Il obtient finalement le poste de courtier en 1769 ; la fonction de «chef des noirs ", en 1773 ; la confirmation de ses fonctions par un ordre royal, en 1776 et un certificat du gouverneur Bellecombe en 1778. Suite à la reddition de la ville la même année et au départ du gouverneur, il perd le bénéfice de ses fonctions. Il peine ensuite à retrouver son statut : il multiplie à nouveau les démarches entre 1785 et $1788^{16}$. La parcellisation des fonctions du dubash en chef peut résulter du jeu des partis. La distribution des postes nouvellement créés serait alors un moyen d'apaiser les tensions dans l'exercice du gouvernement.

14 Les dubashes mobilisent liens personnels et réseaux relationnels pour accéder à des fonctions fortement concurrencées. Ils cherchent également à valoriser leur expertise dans les domaines où les Français expriment leurs attentes ou leurs ambitions, ou imaginent et se représentent leur implantation coloniale. Ils mettent en valeur leur expertise linguistique réelle - langues locales (tamoul, telugu), langue diplomatique (persan), lingua franca du commerce et des échanges avec les Européens (portugais) ou attendue : selon Ananda Ranga Pillai, son neveu Tiruvengadam parlerait le français depuis l'âge de douze ans. Or, lorsque la langue utilisée dans les échanges avec les Français est mentionnée dans le journal de ce dernier, il s'agit du portugais.

15 Les dubashes apparaissent alors comme les acteurs d'un mode de fonctionnement et de représentation de l'empire français en Inde. Cela est particulièrement visible dans le domaine de la diplomatie. Sous le gouvernorat de Dupleix, Ananda Ranga Pillai insiste 
dans son journal sur son expertise diplomatique et sa capacité à mener des négociations fructueuses, faisant écho aux aspirations expansionnistes du gouverneur. L'investissement des dubashes dans les projets coloniaux français concourt à façonner ces projets. Après le rappel de Dupleix, les discours de combats triomphants tendent à disparaitre du journal d'Ananda Ranga. En effet, les efforts diplomatiques n'ont plus alors pour objectif la conquête territoriale. Ils visent plutôt, dans un contexte de conflits réguliers avec l'Angleterre, à contracter des alliances régionales nécessaires au maintien d'une présence française dans le sous-continent. Parallèlement, les discours produits par les acteurs engagés sur le terrain nourrissent le mythe d'une vocation impériale de la France en Inde. Ce mythe repose sur celui de l'action des représentants français auprès des cours princières, entretenu par les courriers et mémoires adressés au ministère de la Marine ${ }^{17}$. Les discours produits par les agents indiens ont pu agir en ce sens. Le journal de Tiruvengadam III rend compte de la manière dont le gouverneur Law de Lauriston, qui l'a écarté du gouvernement, se tourne vers lui lorsqu'il perçoit une opportunité diplomatique dans son réseau de correspondants. L'efficacité diplomatique des dubashes, effective, représentée ou imaginée, constitue un élément clé de leur carrière et un des principaux domaines dans lesquels ils entrent en concurrence.

\subsection{Mises en concurrence : un équilibre des intérêts ?}

16 La concurrence entre les agents indiens relève également d'une mise en concurrence par l'administration coloniale. L'attribution d'un poste clé, convoité et toujours longuement négocié, est un moyen de rétribution et de promotion de l'agent efficace. Cette promotion concerne l'agent mais également sa parentèle. En effet, le bénéfice de la médiation fructueuse s'étend de l'individu au groupe. L'agent cherche à utiliser sa position pour placer les membres de son groupe familial dans l'administration. Pour l'administration coloniale, la mise en concurrence est un moyen de s'assurer des compétences. A une époque où aucune réglementation n'est fixée, où les Français maîtrisent très mal les pratiques sociales locales, la distribution endogène des fonctions fournit des garants pour la nouvelle recrue. Le système concurrentiel repose donc sur un équilibre entre les intérêts du gouvernement et ceux de ses agents indiens. Pourtant, les Français restent maîtres du jeu de balance. Ils veillent à entretenir l'émulation et à accommoder les différentes ambitions, afin, d'une part, de s'assurer de la performance de leurs agents et, d'autre part, de ne pas accorder trop de pouvoir à un seul groupe familial. Un épisode du journal d'Ananda Ranga Pillai permet de mieux saisir ce jeu constant d'équilibre. En janvier 1756, à la mort de son neveu, dubash du tribunal de la Chaudrie, Ananda Ranga Pillai cherche à garder la maîtrise de cette charge, en la présentant comme une prérogative familiale au gouverneur de Leyrit :

17 Je ne suis pas sorti ces trois derniers jours en raison de la mort de Chiranjivi Tambi Venkatachalam $^{18}$. Aujourd'hui, en chemin pour le Fort, j'ai rendu visite à M. Barthélémy ${ }^{19}$ pour le remercier de ses condoléances. Je lui dis «La charge de Venkatachalam devrait être reprise par quelqu'un de ma famille. Je vous prie de donner des ordres allant en ce sens. »M. Barthélémy répondit qu'il donnerait la charge à quiconque je lui proposerais, mais ajouta «Nallatambi était poligar ${ }^{20}$, mais se trouve désormais en grandes difficultés, sans office. Il mendie le poste, et comme il meurt de faim, il devrait être fait poligar quelque part. Le gouverneur a dit qu'il verrait cela, mais j'ai dit à M. Leyrit que le poste a depuis longtemps été tenu par votre famille. Vous 
devriez lui en parler. » Je lui dis que je le ferai, et ayant pris congé de M. Barthélémy, je gagnai le Fort. Le Gouverneur s'entretenait avec plusieurs personnes, et mit beaucoup de temps à rejoindre son bureau, mais j'attendis pour lui présenter mes respects. Je lui dis ensuite: «depuis l'époque de M. Lenoir, pendant 25 ans, Tambi Venkatachalam a été le dubash de la Chaudrie ; quand il est tombé malade, M. Dupleix m'a laissé nommer le fils de mon frère aîné, qui a occupé le poste jusqu'à ce qu'il plaise à Dieu de mettre fin à ses jours. Avec votre permission, je nommerai maintenant le mari de la plus jeune des filles de mon frère. » Le Gouverneur dit qu'il veillerait à ce que le poste soit donné à l'un de mes gens, et qu'il donnerait des ordres en ce sens après avoir parlé à $M$. Barthélémy demain ${ }^{21}$.

18 Ananda Ranga Pillai cherche donc à patrimonialiser la fonction de dubash de la Chaudrie. Ce type de tentative de patrimonialisation des fonctions n'est propre ni à la famille Pillai ni à la situation coloniale. C'est un moyen de court-circuiter les concurrences et de répondre à l'instabilité des positions. Ce mouvement, qui se rencontre tout au long du XVIII e siècle, est renforcé par les difficultés du comptoir dans la seconde moitié du siècle. Une partie des opportunités économiques disparaît avec l'effondrement du commerce ; la baisse des revenus et le manque de moyens entraînent la suppression d'une partie des postes d'agents locaux; après 1793, le commandant britannique de Pondichéry réduit encore l'administration de la ville occupée.

\subsection{Une méfiance croissante}

19 La mise en concurrence produit un double mouvement de mise en valeur de sa propre médiation et de dénigrement de celle des agents rivaux. Tiruvengadam III pointe régulièrement du doigt les abus et la corruption du courtier en chef Candapa. Il estime que ce dernier agirait suivant ses intérêts et non selon les instructions du gouverneur, qualifié de "marionnette ${ }^{22}$.» Ce type d'accusations visant à décrédibiliser le parti adverse apparait dans la documentation depuis la fondation du comptoir, constituant une sorte de topos partagé par les agents français et indiens. La défiance, constitutive $\mathrm{du}$ système relationnel dans l'espace du comptoir, prend toutefois une dimension nouvelle dans la seconde moitié du XVIII ${ }^{e}$ siècle.

20 La diffusion des représentations forgées à partir des années 1740 par les philosophes français participe de l'évolution des conceptions de l'administration politique de l'Inde ${ }^{23}$. La définition progressive d'une identité européenne des Lumières, moderne et éclairée, construit en contrepoint une identité orientale vénale, fastueuse et despotique ${ }^{24}$. Les pratiques jusqu'ici reconnues comme parties intégrantes des systèmes politiques locaux sont désormais regardées comme déviantes, et déconsidérées. En février 1765 , le capitaine de vaisseau Surville fait remarquer à Tiruvengadam III que son oncle Ananda Ranga Pillai «a dépensé d'importantes sommes d'argent et vécu dans la pompe et la splendeur. On ne devrait pas dépenser autant d'argent pour le service de la Compagnie. Les occasions se présentent souvent, où l'on ferait mieux de se montrer discret et frugal. Les temps anciens, de toute manière, ont changé, et les anciens usages ont disparu ${ }^{25}$." Les agents français invitent à la «frugalité » des pratiques. Parallèlement, ils cherchent à encadrer ces pratiques. Alors qu'au début du siècle il était considéré comme normal de "faire de l'argent ${ }^{26}$ ", à partir des années 1750 , la Compagnie tente de limiter les épices et la concussion. Elle interdit à ses employés de participer personnellement aux processus politiques reposant sur l'échange de cadeaux. 
Elle annule une partie des donations faites aux gouverneurs et ordonne une enquête sur les comptes de Dupleix ${ }^{27}$.

21 Une distinction est opérée entre l'individu et la fonction qu'il occupe. Ce n'est plus le gouverneur, en tant que personne, qui peut recevoir et s'approprier des cadeaux, mais le gouverneur en tant que représentant de la Compagnie. Cette distinction concerne tous les agents, français ou indiens, et va de pair avec un effort législatif de formalisation et de normalisation des statuts et des pratiques ${ }^{28}$. La parcellisation des fonctions du courtier en chef, en 1769, pourrait s'inscrire dans cette dynamique et être une manière de mieux maîtriser des fonctions qui jusque-là relevaient largement de rapports interpersonnels. Les représentations des dubashes évoluent avec les conceptions de la gouvernance, et l'administration se méfie davantage de l'expertise stratégique de ses agents, qui leur confère un pouvoir menaçant ses intérêts. Dans les années 1770 , des administrateurs français documentent leurs griefs contre le courtier en chef Candapa. Il est accusé de corruption, de fraudes dans les fermes et de spéculations sur le grain, qui nuisent à l'approvisionnement du comptoir. L'ordonnateur de Courcy, en 1776, dénonce les « manœuvres secrètes » de cet « homme fin et rusé ", " qui ne s'occupe qu'à détruire [les intérêts de sa Majesté] »" ${ }^{29}$. Il diffuse l'image du dubash immoral et puissant, agissant de manière souterraine, tandis que l'intermédiaire loyal - Tiruvengadam III - ne trouverait plus sa place dans l'administration du comptoir. Les menées contre les agents indiens se poursuivent lors de la période révolutionnaire, avec le scandale de la liquidation de la seconde Compagnie des Indes; après 1793, les administrateurs à Pondichéry portent des plaintes au tribunal de la Chaudrie contre Naynatté Modely et Visea Raya Modély, les deux principaux marchands et intermédiaires de la Compagnie ${ }^{30}$.

Ce type d'accusations s'inscrit dans une évolution générale de la conception des intermédiaires dans les espaces dominés. Le même processus se retrouve dans les possessions britanniques. Susan Neild-Basu relève la multiplication des plaintes et des procès contre les dubashes de Madras. Alors que l'East India Company renforce sa gouvernance, le sentiment de défiance s'accentue, à la fin du siècle, vis-à-vis d'hommes qu'elle estime avoir construits. Ils lui devraient tout, mais possèderaient désormais, grâce à elle, une autorité propre et dangereuse ${ }^{31}$. Dans leur introduction, les éditeurs de The Brokered World repèrent l'apparition du terme go-between à la même époque, dans les écrits des agents de l'EIC ou ceux d'hommes politiques anglais comme Edmund Burke, pour désigner l'intermédiaire indien dont les attributions multiples en font un agent indispensable, mais peu fiable ${ }^{32}$. Le go-between est alors une figure construite par l'interaction: il est l'intermédiaire tel que représenté par les individus ou les institutions qui bénéficient de sa médiation.

\section{Stratégies de sécurisation du statut : l'usage de l'écrit entre innovations et ancrage dans les pratiques locales}

Les dubashes mettent en place différents types de stratégies pour s'adapter aux mouvements de l'administration, valoriser leur expertise et sécuriser leur position. Nous nous intéresserons ici aux stratégies liées à l'écrit, c'est à dire à la production de documents de natures diverses, par ou pour les dubashes. 


\subsection{Les certificats produits par l'administration française : dire l'utilité des dubashes}

24 Les certificats sont produits dans, par et pour les interactions avec les administrateurs français. Plusieurs exemples montrent que les certificats sont demandés par les dubashes à l'administration coloniale. Il s'agit de textes brefs rédigés par l'autorité de référence, sur papier, attestant du statut et / ou des services rendus par les dubashes. Trois certificats sont remis après les sièges de Pondichéry aux membres de la famille Pillai, soulignant leur "utilité». Ananda Ranga Pillai fournit du riz en 1760, Tiruvengadam III recrute et finance une troupe de soldats en 1778 et Tiruvengadam IV se montre d'une "grande utilité » en $1793^{33}$. Le rapport d'utilité est réciproque : les Français ont besoin de l'engagement matériel de leurs agents, en particulier en temps de crise, et ces agents ont besoin des Français pour garantir leur statut économique et social. Cette forme d'équilibre des intérêts ne signifie pas qu'il y a égalité dans l'interaction : l'arbitraire des Français, les scandales et les rapports de domination sont des constantes des relations interculturelles à Pondichéry. Mais chaque partie mesure les avantages, les inconvénients et les risques de l'interaction ${ }^{34}$.

Les certificats sont destinés à être produits pour appuyer une demande de poste. Ils sont adressés au gouverneur du comptoir, voire, dans le cas des démarches des membres de la famille Pillai, aux différents niveaux hiérarchiques (conseillers, administrateurs, ministre de la Marine, roi de France). Tiruvengadam III et Tiruvengadam IV obtiennent de l'administration coloniale dix certificats garantissant leur fonction de chef des Indiens ou de courtier en chef, entre 1773 et $1793^{35}$. Dans le cas de ce groupe familial, le rapport à l'écrit n'est pas un phénomène nouveau. Ce qui est nouveau, c'est la multiplication des certificats dans le dernier tiers du siècle, demandés ou produits par divers agents indiens. Ce mouvement rend compte de la compréhension de l'évolution des rapports interpersonnels comme forme de garantie, et de l'autorité nouvelle du document écrit et signé par l'administration coloniale ${ }^{36}$. La demande de recommandations écrites est un moyen de sécuriser les statuts en période d'incertitudes et d'imprévisibilités : Pondichéry est prise et occupée trois fois en trente ans, ce qui entraîne pour les agents indiens des pertes matérielles lourdes et un éclatement des réseaux de clientèle - avec, souvent, le départ des protecteurs français. Les certificats sont alors destinés, en l'absence de relais, à être transmis aux nouveaux administrateurs, qui dans le dernier quart du siècle sont parfois peu au fait des aménagements et des équilibres locaux. L'objet-papier est un marqueur de la confiance passée et appelle en retour la confiance des nouveaux agents français.

\subsection{Mémoires et journaux : représenter l'ancienneté de la médiation}

Lorsqu'il adresse une demande à l'administration, Tiruvengadam III accompagne les certificats de "Mémoires ", constitués d'une douzaine de feuillets, retraçant le rôle de ses ancêtres à Pondichéry ${ }^{37}$. Il ne les présente pas comme de simples intermédiaires au service de la Compagnie, mais comme de véritables acteurs de la politique française en Inde, de grands hommes faisant bénéficier le gouvernement de leur expertise et de leurs réseaux personnels : son grand-oncle Naniapa et son grand-père Tiruvengadam I 
auraient par exemple fait passer Pondichéry du rang de village à celui de place commerçante :

[La ville] n'était qu'une bourgade, et à proprement parler qu'un bois couvert de palmiers et de mauvais arbres sauvages, dépourvue de toute sorte d'ouvriers et d'indigents et en tout peuplée seulement que de quelques petits marchands, la plupart boutiquaires et autres malabars occupés à l'agriculture des fruits de laquelle ils vivaient sans aucun autre commerce. Tirouvengadanpoullé, de concert avec Nainapapoullé [...] écrivirent à leurs correspondants dans les villes et aldées de cette province lesquels envoyèrent des marchands, des tisserands, des peintres en toile, des ouvriers de toute sorte de métiers et de professions alors la colonie commença à prendre un certain lustre, l'on vit fabriquer et peindre des toiles et le commerce s'ouvrit tant par mer que par terre ${ }^{38}$.

Le récit de l'engagement à la fois fructueux, ancien et constant au service des Français se retrouve dans les journaux d'Ananda Ranga Pillai et de Tiruvengadam III, en particulier lorsqu'ils rapportent les propos qu'auraient tenus leurs interlocuteurs français. Ces dubashes font ainsi de la fidélité de leur famille un motif central de leur narration, à une période où les changements d'alliance ou d'allégeance sont constants et jouent à différentes échelles - locales, régionales, impériales ${ }^{39}$. A travers leurs choix narratifs, ils définissent un modèle représentationnel. Simon Schaffer a bien relevé que les intermédiaires actifs à la fin du XVIII ${ }^{e}$ siècle dans les réseaux commerciaux n'avaient pas seulement exercé leur métier de marchand en tant qu'intermédiaires, mais avaient également activement et intelligemment théorisé le rôle de go-between ${ }^{40}$. Ananda Ranga Pillai et son neveu théorisent ainsi leur rôle et mettent en scène la juste place qu'ils entendent occuper dans la société coloniale. Une partie des études portant sur le journal d'Ananda Ranga Pillai a souligné son caractère personnel et intime, mais il est nécessaire de continuer à s'interroger sur sa nature et sa finalitét ${ }^{41}$. On peut l'envisager comme un document programmatique, définissant l'intermédiaire idéal, valorisant sa loyauté et la validité de son expertise, et destiné à être porté à la connaissance des administrateurs du comptoir ${ }^{42}$. Les propos que le courtier prête régulièrement aux administrateurs auraient alors un caractère performatif, validant et ancrant le statut acquis à travers l'interaction avec les Français.

\subsection{Poèmes sanskrits et tamouls : inscrire l'histoire de la famille dans la société indienne}

29 La question de la validation d'un statut économique et social acquis dans l'interaction avec les Européens est cruciale. L'historienne Kanakalatha Mukund établit que les dubashes actifs à Madras et à Pondichéry ne font pas partie des élites traditionnelles. Ils appartiendraient plutôt à des familles de marchands, auxquels les villes portuaires européennes offrent des «boulevards » pour se forger un statut et constituer une classe d'élites nouvelles ${ }^{43}$. La famille Pillai n'appartient effectivement pas aux hautes castes traditionnelles tamoules, comme les Vellajas, mais à la caste pastorale Idayiar. Il est significatif que la mention de la caste, généralement présente dans les requêtes produites par les Indiens, n'apparaisse pas dans les documents de la famille ${ }^{44}$.

Les Européens fournissent les opportunités nécessaires à la construction d'un statut nouveau, mais ce statut est défini, fondamentalement, par l'ancrage dans les pratiques des élites locales. A aucun moment, les Français ne constituent une référence ou un 
modèle. Les journaux des courtiers montrent leur peu d'intérêt pour les usages culturels français - alors qu'Ananda Ranga Pillai se met régulièrement en scène expliquant les coutumes tamoules à ses interlocuteurs français. Le modèle que ces nouveaux notables cherchent à imiter est celui des anciennes élites foncières, ou de la royauté. Elles reproduisent leurs normes et prennent en charge leurs attributions, comme le patronage des arts et des institutions religieuses. La famille Pillai entretient plusieurs chaudries (lieu d'accueil des voyageurs et d'une communauté de brahmanes) et Ananda Ranga Pillai est un grand patron des arts. Plusieurs poèmes en tamoul ou en sanskrit lui sont dédiés, dans lesquels il est représenté suivant les normes de ces genres littéraires ${ }^{45}$. Dans le poème Ananda Ranga Campu, écrit en sanskrit et présenté en 1752, il apparaît comme un prince pieux et vertueux, à l'ascendance divine, menant les campagnes des Français, chevauchant à la tête des armées, suscitant le respect des soldats et l'adoration de la foule, s'adressant en égal à Dupleix qui l'invite à s'asseoir sur un trône pour célébrer sa victoire ${ }^{46}$. Il n'est plus, ainsi que dans le journal, l'agent du gouverneur qui sait si bien tenir sa position, mais son égal royal. La famille cherche à ancrer son identité dans les systèmes culturels locaux, suivant une logique de la distinction : elle se rend reconnaissable aux populations locales en même temps qu'elle établit une différence fondamentale, et non surmontable, vis-à-vis des Européens.

\section{Des intermédiaires indispensables au gouvernement du comptoir}

31 Même si le statut des dubashes nait de l'interaction avec l'administration coloniale, leur appartenance, première et essentielle, est indienne et locale. Leur maîtrise des formes culturelles propres aux différentes sociétés indiennes constitue un des éléments-clés de leur expertise. Ils restent des intermédiaires indispensables au gouvernement $d u$ comptoir, quelle que soit la situation économique ou militaire.

\subsection{Une maîtrise des formes culturelles nécessaire à l'exercice de la souveraineté}

Dans les situations de rencontre, d'échange ou simplement de coprésence, les dubashes disent et traduisent les formes et les normes culturelles et sociales qui participent de l'exercice de l'autorité souveraine. Ils positionnent le gouvernement de manière appropriée dans les relations sociales et culturelles, garantissant son prestige, et, partant, sa légitimité ${ }^{47}$. En participant eux-mêmes à ce système, ils renforcent leur prestige personnel et celui de leur groupe familial. Leur médiation est alors un mélange de décryptage et de prise en charge directe des rapports interculturels. Ils maitrisent et traduisent le protocole et le cérémonial de cour, adoptés par l'administration coloniale. Ainsi que l'explique Sanjay Subrahmanyam, la connaissance exacte de l'étiquette et du comportement adéquat d'Ananda Ranga Pillai est «cruciale dans les situations délicates d'interaction interculturelle ${ }^{48}$. » Ananda Ranga se met régulièrement en scène traduisant le sens de pratiques ou de postures, et dictant au gouverneur l'attitude à adopter, par exemple ici après une rencontre avec un groupe de cavaliers :

[Dupleix] me dit avec aigreur : « comme j'étais à cheval, le fils de Chanda Sahib et cinq ou six cavaliers allaient voir la statue qu'on portait en procession. Ils m'ont tourné le dos. Comment se fait il qu'ils m'aient manqué à ce point de respect? »[...] Je lui dis : 
"Chez les Musulmans, quand on rencontre son père, sa mère ou quelqu'un de plus âgé que soi, il n'est pas convenable de lui faire face, mais l'on doit s'arrêter et s'écarter. C'est aussi l'usage chez les Tamouls. Comme Raza Sahib vous regarde comme son père, il s'est immobilisé et tenu à l'écart. - En ce cas, tout est pour le mieux, fit-il ${ }^{49}$.

Le courtier ordonne (met en ordre et commande) les formes culturelles. Il identifie et définit la place de chacun, par exemple, nous l'avons vu, l'ordre dans lequel les élites indiennes doivent être présentées au gouverneur. C'est lui qui organise les festivités qui encadrent les échanges diplomatiques - danseuses, musiciens, salves, feux d'artifice, pompes, parades, etc. Il est également celui qui reçoit, aux limites de la ville, les présents destinés au gouvernement, ou qui détermine, selon le statut de chacun, la valeur des présents et des honneurs à distribuer. La distribution des honneurs rituels est un mode d'exercice de l'autorité politique : prise en charge par le prince - et donc, dans le contexte de la ville coloniale, par le gouvernement européen, elle valide le statut des individus et des groupes. Les agents indiens recherchent les privilèges et les insignes honorifiques, Ananda Ranga Pillai le premier : dans son journal, il fait grand cas des médailles offertes par le gouvernement, affirme être le seul Indien autorisé à circuler dans l'enceinte du fort en palanquin, ou précédé du roundel (grand parasol blanc).

L'administration coloniale fait le choix d'assumer les attributs du prince. Dans l'exercice de la souveraineté, elle compose avec les pratiques locales et ses propres possibilités. Confrontée à un manque chronique de moyens, elle utilise la distribution d'honneurs comme un moyen de rétribution de ses agents. Lorsque le Conseil supérieur de Pondichéry crée une chambre de consultation de jurisprudence indienne en janvier 1778, pour conseiller les juges français, il ne prévoit pas de traitement pour ses membres, mais proclame qu'«ils seront considérés comme principaux habitants et jouiront des prérogatives accordées à l'importance de leurs fonctions, telles que: maniam et droit de porter la médaille qui leur sera donnée par Messieurs les Administrateurs au nom de Sa Majesté ${ }^{50}$.»

\subsection{Le choix de la délégation}

La chambre de consultation s'inscrit dans un mode spécifique de fonctionnement et de représentation du gouvernement colonial. Du début du XVIII ${ }^{\mathrm{e}}$ siècle au milieu du XIX ${ }^{\mathrm{e}}$ siècle, les Français proclament à plusieurs reprises leur engagement à respecter les "us et coutumes " des Indiens, c'est-à-dire tout ce qui se rapporte à la caste, aux pratiques religieuses, et aux questions de parenté, de maritalité, et de succession. Le choix du respect des «coutumes" et de la prise en charge des "traditions» locales comme moyen de gouvernement se retrouve, à différentes échelles, dans la plupart des sociétés coloniales ${ }^{51}$. A Pondichéry à la fin du XVIII ${ }^{\mathrm{e}}$ siècle, ce choix est influencé par les savoirs orientalistes : de même que les Britanniques, les Français imaginent la société indienne comme antique et statique, gouvernée par des institutions et des lois ancestrales, pour laquelle les « us et coutumes » fonderaient les rapports sociaux et le mode de règlement des conflits ${ }^{52}$. Tout au long de la période, ce choix est surtout un choix pragmatique, répondant à des situations locales. Les administrateurs soulignent l'agitation des populations hindoues en cas d'atteinte portée à leur culte, et les tensions de la première moitié du siècle, montrent que le rapport de force n'est pas toujours en faveur du colonisateur ${ }^{53}$. Les possibilités très limitées de l'administration coloniale rendent nécessaires la délégation à des institutions et des agents indigènes. Outre la 
faiblesse des moyens matériels et l'insuffisance de personnel, la méconnaissance des langues vernaculaires joue un rôle important dans ce processus. On ne repère pas, en effet, dans le cas français, la dynamique à l'œuvre dans l'empire britannique, à partir des années 1770-1780, d'apprentissage des langues locales. De même, les efforts de compilation des savoirs pratiques et juridiques restent très partiels jusqu'aux années 1820 , et le projet de rédaction d'un ouvrage de législation et de jurisprudence confié à la chambre de consultation en 1778 n'aboutit pas ${ }^{54}$.

Les dubashes sont donc indispensables à l'administration française parce qu'elle les institue comme tels. Elle fait le choix de l'utilité des relais: soldats, agents de police, principaux dubashes de l'administration, et personnel judiciaire. Le rôle du personnel judiciaire est particulièrement important en raison de l'organisation duale de la justice dans les comptoirs français de l'Inde: le Conseil supérieur juge les affaires entre Européens et le tribunal de la Chaudrie les affaires entre Indiens ou entre Indiens et Européens ${ }^{55}$. A l'exception des cas relevant des «us et coutumes ", les conflits entre Indiens portés devant la justice coloniale sont donc essentiellement examinés à la Chaudrie. Les juges et les assesseurs de ce tribunal sont des membres du Conseil supérieur, sans formation juridique spécifique. Régulièrement, ils s'en remettent à l'arbitrage des autorités de parenté ou de caste pour les affaires familiales, ou entérinent la décision d'arbitres choisis par les parties. Le processus de délégation fonctionne également en interne: l'instruction des affaires peut être confiée à l'interprète ou à l'huissier indien du tribunal, qui sont alors investis d'une autorité importante sur la société locale. L'autonomie et l'influence des intermédiaires du système judiciaire sont réelles; leur décision est presque systématiquement sanctionnée. Mais cette autonomie et cette influence ne sont pas reconnues en tant que telles: les postes les plus importants, impliquant la prise de décision ou la validation finale de la décision, sont réservés aux Français. Le pouvoir judiciaire et la capacité de contrainte sur les individus, leurs corps et leurs propriétés, apparaissent comme des prérogatives du gouvernement.

La justice et le règlement des conflits constituent un terrain privilégié pour examiner les pratiques de l'administration coloniale et sa conception de la souveraineté. Cette conception accommode celle de l'Etat dans la France moderne, les pratiques des souverains du sud de l'Inde et les contraintes liées au contexte local. Ainsi, les conflits de caste, définis comme relevant de l'ordre public, doivent être portés à la connaissance du lieutenant de police et des administrateurs, et tranchés par le gouverneur, qui conserve le pouvoir décisionnaire tout au long de la période, malgré des ajustements de procédure. La connaissance de ces conflits, pourtant, semble pouvoir être également déléguée au chef des Malabars ${ }^{56}$.

\subsection{Une institution spécifique : le chef des Malabars}

Il est difficile de définir précisément les prérogatives du chef des Malabars. Ce titre apparaît peu dans la documentation, avant d'être mobilisé au milieu du siècle par Ananda Ranga Pillai, qui l'investit d'une charge considérable. À l'occasion du récit de son « investiture » comme courtier en chef, le 2 janvier 1755, il rapporte les paroles qu'aurait tenues le gouverneur Godeheu: "J'ordonnerai à tous ceux qui ne sont pas Européens, à savoir les Tamils, les Musulmans et tous les Indiens, de vous obéir comme à leur maître. » Aux notables tamouls venus lui présenter leurs vœux, Godeheu aurait 
déclaré : «A partir de ce jour, vous n'aurez plus affaire directement à moi, mais vous irez trouver Ranga Pillai et exécuterez ses ordres. Quiconque ne le fera pas encourra une amende ou d'autres punitions ${ }^{57}$. " Il faut s'interroger sur la stratégie discursive d'Ananda Ranga : un tel poste, équivalent de celui de gouverneur pour la population indienne, conférerait une autorité autonome sur une population cinquante fois plus nombreuse que la population française, et représenterait un danger sérieux pour l'autorité coloniale ${ }^{58}$. Un autre document de la famille Pillai identifie de manière similaire les prérogatives du chef des Indiens. Il s'agit d'un certificat qui aurait été délivré en 1785 à Tiruvengadam par le gouverneur de Cossigny et l'ordonnateur Moracin, et confirmé par le gouverneur général de Souillac ${ }^{59}$ :

Le dit Rangapa Tirevengadon continuera d'être chef des indiens et malabars comme il l'était avant la reddition de cette ville en 1778. A ce titre, nous lui attribuons toute autorité et inspection sur tous lesdits indiens et malabars, auxquels nous ordonnons respect et obéissance envers le dit Tirevangadon; nous autorisons nommément ce dernier à recevoir, écouter, discuter et nous porter les plaintes ou griefs que les dits indiens et malabars pourraient avoir entre eux, pour y être par nous fait tel droit que nous aviserons bon être suivant l'exigence des cas; nous attribuons plus particulièrement encore au dit Rangappa Tirevangadon le droit exclusif de connaître seul de toutes les disputes ou discussions entre la main droite et la main gauche, lui recommandant de les prévenir autant que possible et de les faire cesser quand elles seront avenues ${ }^{60}$.

La Main Gauche et la Main Droite constituent les deux grandes divisions au sein desquelles les castes sont réparties dans une partie de l'Inde du sud. Ces deux divisions correspondent à deux grands partis qui s'affrontent, de manière violente, pour l'obtention de privilèges, de droits ou d'insignes honorifiques, comme le port d'étendards ou l'utilisation de palanquins ou de chevaux lors des cérémonies. Niels Brimnes a analysé comment les Européens, assumant au XVIII ${ }^{e}$ siècle le rôle du " petit roi", sont mobilisés pour la concession de privilèges ou l'interdiction de toute appropriation de ces privilèges par le parti rival ${ }^{61}$. A Pondichéry, comme dans le comptoir britannique de Madras et le comptoir danois de Tranquebar, l'opposition violente des deux Mains a pour but de forcer l'autorité européenne à se positionner. Les Européens restent très réticents à intervenir, mais y sont contraints lorsque des émeutes particulièrement graves menacent l'ordre de l'établissement.

A Pondichéry, les conflits les plus violents ont lieu en 1768, 1776 et $1785^{62}$. En 1768, le gouvernement français assume la résolution du conflit entre les Mains et s'affirme comme le garant de l'ordre public qu'il a défini. En 1776 et 1785, il délivre des certificats à Tiruvengadam, lui attribuant le titre de chef des Malabars et des pouvoirs étendus. Il est très probable que l'administration française ait effectivement tenté de déléguer à Tiruvengadam III les pouvoirs énumérés dans le certificat de 1785. A cette date, après dix-neuf mois de négociations avec l'Angleterre, la France vient de reprendre possession de Pondichéry, mais se désintéresse de ses fragiles établissements indiens. Ils sont réunis aux Îles de France (Maurice) et de Bourbon (Réunion) dans un gouvernorat général dont le siège est à Port Louis. L'administration des comptoirs est ralentie par la distance du centre décisionnaire et par l'aggravation du manque déjà chronique de moyens. Les réformes enclenchées dans les années 1770 sont abandonnées, en particulier les tentatives de réorganisation de la justice - on se repose désormais sur des juges notables. Le gouvernement colonial pourrait alors avoir investi 
un intermédiaire indien d'un pan de l'autorité souveraine, afin de réguler des conflits qu'il n'était pas capable de maîtriser. Cela expliquerait l'intérêt majeur de Tiruvengadam III pour la fonction de chef des Indiens, qui ne semble exister ni à Madras ni à Tranquebar. L'intermédiaire serait alors placé à la fois dans la position d'arbitre et de tertius gaudens, au sens défini par Georg Simmel : un acteur dont le rôle repose sur la confiance des parties, de manière à éviter l'éclatement du groupe l'arbitre ; et un tiers qui tire profit de la situation conflictuelle entre deux parties ${ }^{63}$.

Quelles que soient l'effectivité et l'efficacité de la délégation de pouvoir au dubash, cette attribution est révélatrice des tensions à l'œuvre dans l'exercice de la souveraineté. Le gouvernement colonial renonce à exercer une partie de ses prérogatives lorsqu'il n'en a plus les moyens. Ces prérogatives intéressent les dubashes. Le jeu des intérêts s'équilibre lorsque le comptoir connaît ses épisodes les plus difficiles, et la délégation de la souveraineté s'accroît avec la précarité des établissements français de l'Inde. Les modalités d'exercice de la souveraineté sont donc évolutives. Elles fluctuent également au gré des tentatives de réforme et de formalisation de l'administration, ou de la personnalité des administrateurs. Le règlement de police de 1778, qui ne sera jamais appliqué en raison de la prise de Pondichéry, attribue l'instruction des affaires de caste au lieutenant de police, en lien avec les administrateurs, alors que les certificats de 1773, 1776, 1777 et 1785 délèguent la connaissance de ces affaires au chef des Malabars $^{64}$. Un principe directeur se maintient: la délégation de la souveraineté s'organise de manière duale ; elle est distribuée aux élites des deux grandes catégories de population: européenne et indienne. La logique impériale de différenciation dans l'administration des populations reste l'œuvre tout au long du siècle ${ }^{65}$. Elle pose problème à partir de 1790 et de l'organisation des citoyens de Pondichéry en comité représentatif puis en municipalité. Pourtant, la catégorisation des populations et la politique de distinction, contestées et débattues, se maintiennent au cours de la période révolutionnaire ${ }^{66}$.

\section{Conclusion}

La prise en charge des systèmes culturels et sociaux locaux, nécessaire à l'exercice et au maintien de l'autorité coloniale, ne peut se faire sans la médiation d'agents indiens. Les dubashes ont ainsi un rôle central à Pondichéry dans la seconde moitié du XVIII ${ }^{\mathrm{e}}$ siècle. Ce rôle leur permet de construire et de renforcer leur statut économique et social. Il permet parallèlement au gouvernement français de s'assurer de la légitimité de sa souveraineté, et d'une forme de domination locale non conflictuelle. Les relations entre l'administration française et ses principaux agents indiens s'articulent autour de rapports d'intérêt et d'utilité réciproques. Ces rapports sont particulièrement visibles dans le jeu des concurrences pour accéder aux principaux postes de médiation. Pour répondre à cette concurrence et à la méfiance croissante de la faible administration coloniale, les dubashes de la famille de Tiruvengadam mettent en place différentes stratégies liées à l'écrit. La production de mémoires et de certificats reflète la médiation fructueuse, ancienne et privilégiée. En même temps, le patronage de la poésie tamoule et sanskrite dit la différence fondamentale et non surmontable vis-à-vis des Français. Cette maîtrise des formes et des codes culturels participe de l'utilité des dubashes, telle qu'elle est perçue et en même temps construite par le gouvernement colonial. Ce dernier fait le choix de la délégation d'un certain nombre de domaines d'actions et de 
compétences à ses agents indiens, allant jusqu'à attribuer une partie de sa souveraineté au Chef des Malabars.

Dans le cas du règlement des conflits entre castes ou entre Mains, et, de manière plus large, dans un grand nombre de domaines de la vie sociale, la gestion de la différence passe par des arrangements avec les élites locales. L'« intégration » de ces élites est un outil de la politique de "différenciation» du gouvernement colonial ${ }^{67}$. Les agents indiens sont "intégrés» dans le sens où ils font partie et participent du fonctionnement de l'administration (ils reçoivent par exemple un traitement porté sur les états des dépenses), mais il n'y a pas d'incorporation. On observe plutôt, ici encore, un ensemble de relations fluides et flexibles, de participation et d'intégrations partielles reposant sur des rapports d'intérêt.

Ces rapports d'intérêt nourrissent la concurrence entre les dubashes, mais également la compétition avec d'autres groupes d'intermédiaires spécialisés, comme les marchands de la Compagnie française des Indes au milieu du siècle. Les tensions entre les dubashes et les habitants indiens de Pondichéry apparaissent en revanche très peu dans la documentation. Elles concernent essentiellement des affaires privées (commerce, propriété foncière), ou des abus dans la gestion des fermes des terres ou des revenus. Ces traces dans les archives de l'administration coloniale peuvent permettre de comprendre les formes de mobilisation des populations locales au sein de la société coloniale, et notamment le rôle des institutions intermédiaires (assemblées de parenté, de caste ou de village) avec lesquelles les dubashes sont en relation.

\section{NOTES}

1. S. Jeyaseela STEPHEN (dir.), The Diary of Rangappa Thiruvengadam Pillai, 1761-1768, Pondicherry, IIES Publisher, 2001, p. 255.

2. Georg SIMMEL Sociologie. Etudes sur les formes de la socialisation, Paris, PUF, 1999 (1908) ; pour une présentation synthétique, voir Irwin PRESS, « Ambiguity and Innovation: Implications for the Genesis of the Cultural Broker ", American Anthropologist, vol. 71, n² 2, 1969, p. 205-217.

3. Clifford GEERTZ, "The Javanese Kijaji: the Changing Role of a Cultural Broker ", Comparative Studies in History and Society, vol. 2, n 2, 1960, p. 228-249.

4. Certains historiens ont produit des travaux précurseurs, notamment Michel Vovelle, qui a organisé un colloque pluridisciplinaire sur les intermédiaires culturels à Aix-en-Provence en 1978, ou Peter Marshall, qui a travaillé sur les banyans de Calcutta dans les années 1970. A partir des années 1990, les historiens travaillant sur l'espace nord-américain se sont intéressés au rôle des agents indiens dans les négociations, les échanges commerciaux et la signature de traités (Richard WHITE, Le Middle Ground. Indiens, empires et républiques dans la région des Grands lacs, 1650-1815, Toulouse, Anacharsis, 2009 (1991); Margaret coNNEL SZASZ (dir.), Between Indian and white worlds: the cultural broker, Norman, University of Oklahoma Press, 1994). Les historiens de l'Amérique latine ont étudié les intermédiaires culturels (Serge GRUZINSKI, Louise BENAT-TACHOT (dir.), Passeurs culturels. Mécanismes de métissage, Paris, Presses Universitaires de Marne-la-Vallée, Éditions de la Maison des Sciences de l'Homme, 2001), ou «transactionnels » (Alida METCALF, Gobetweens and the Colonization of Brazil, 1500-1600, Austin, University of Texas Press, 2005). 
Actuellement, les chantiers de la nouvelle histoire impériale se penchent sur les intermédiaires impériaux (Jane BURBANK, Frederick COOPER, Empires, De la Chine ancienne à nos jours, Paris, Payot, 2011 (2010)).

5. Sanjay subrahmanyam, Explorations in Connected Histories. From the Tagus to the Ganges, Delhi, Oxford University Press, 2004. Les travaux de Sanjay Subrahmanyam rendent compte de la nécessité pour les historiens de maîtriser des langues locales et de connaître la diversité des sources disponibles, en particulier les sources littéraires, afin d'éclairer les parcours des intermédiaires; voir Muzzafar ALAM, Sanjay SUBRAHMANYAM, "The Making of a Munshi», Comparative Studies of South Asia, Africa and the Middle East, vol. 24, n 2, 2004, p. 61-72 ; James ONLEY, "Britain's Native Agents in Arabia and Persia in the Nineteenth Century ", Comparative Studies of South Asia, Africa and the Middle East, vol. 24, n 1, 2004, p. 129-138.

6. Simon SCHAFFER, Lissa RoBerTs, Kapil RAJ, James Delbourgo (dir.), The Brokered World: Go-Betweens and Global Intelligence, 1770-1820, Sagamore Beach, Uppsala Studies in History of Science, $\mathrm{n}^{\circ} 35$, 2009.

7. Sur le XVIII ${ }^{\mathrm{e}}$ siècle, voir les travaux de Jean-Marie Laffont et de Maya Jasanoff pour le rôle des aventuriers français au service des princes indiens à la fin siècle; de Philippe Haudrere pour l'histoire du commerce et de la Compagnie française des Indes; de Siba Pada Sen, Jacques Weber et Massimiliano Vaghi pour l'histoire politique et militaire. Plusieurs recherches sont actuellement en cours et feront l'objet d'un numéro spécial d'Outre-Mers. Revue d'histoire.

8. Ce contexte troublé provoque un effondrement du commerce, notamment du commerce intraasiatique, voir Frédéric MANTIENNE, "Le commerce intra-asiatique français aux XVII ${ }^{\mathrm{e}}$ et XVIII ${ }^{\mathrm{e}}$ siècles ", dans Jacques WEBER (dir.), Les relations entre l'Inde et la France, de 1673 à nos jours, Paris, Les Indes savantes, 2002, p. 63-64. La documentation souligne la baisse des revenus et les difficultés d'approvisionnement du comptoir, voir par exemple Indian National Archives, centre de Lawspeth, série 21, dossier 24 (INAL 21, 24) ; Archives Nationales d'Outre Mer, Aix en Provence (ANOM) Col E 379 Bis ; Un Livre de Compte de Ananda Ranga Poullé, courtier de la Compagnie des Indes, Société de l'Histoire de l'Inde Française, Paris - Pondichéry, Leroux - Bibliothèque Coloniale, 1930.

9. Jane BURBANK, Frederick COOPER, Empires, op. cit., p. 30 ; « Empires, droits et citoyenneté de 212 à 1946 », Annales, Histoire, Sciences Sociales, mai/juin 2008/3, 63 ${ }^{\mathrm{e}}$ année, p. 499.

10. Sanjay SUBRAHMANYAM, «Between a Rock and a Hard Place ", dans Simon SCHAFFER et al. (dir.), The Brokered World, op. cit. Dans la documentation, le terme apparait également sous une forme francisée : « dobachi ».

11. Ils restent des partenaires commerciaux et financiers, et s'intéressent aux fermes des terres et des revenus de la Compagnie. Les historiens ont bien montré l'importance du rôle des intermédiaires indiens, aux XVII ${ }^{\mathrm{e}}$ et XVIII ${ }^{\mathrm{e}}$ siècles, dans l'insertion des compagnies européennes dans des réseaux marchands actifs en Asie et dans l'Océan indien, ainsi que leur accès aux réseaux de crédit indigènes; voir notamment Sinnappah Arasaratnam, Merchants, Company and Commerce on the Coromandel Coast, Delhi, Oxford University Press, 1987.

12. ANOM, Fonds Ministériel, Série Géographique Inde, dossier 379, sous-dossier 420 (ANOM FM SG INDE 379, 420).

13. Kanakalatha MUKUND, The View From Below, New Delhi, Orient Longman, 2005, p. 8-9; Sanjay SUBRAHMANYAM, « Between a Rock and a Hard Place », art. cit., p. 438.

14. Carlo GINZBURG, Rapports de force. Histoire, rhétorique, preuve, Paris, Seuil, 2003 ; Ann Laura STOLER, Along the Archival Grain. Epistemic Anxieties and Colonial Common Sense, Princeton, Princeton University Press, 2009.

15. Dans le cas des gouverneurs qui n'ont pas séjourné en Inde avant leur nomination, la recommandation joue un rôle important. 
16. ANOM Col E 379 Bis; INAL 21, 25; A. KRISHNASAMY PILLAI, «The family of Ananda Ranga Pillai, some genealogical data ", Revue Historique, vol. 10, 1972, p. 21; S. Jeyaseela STEPHEN (dir.), The Diary of Rangappa, op. cit.

17. Alors que l'historiographie a bien montré le caractère éclaté du lien avec les puissances régionales, voir Siba Pada SEN, The French in India : 1763-1816, Calcutta, K. L. Mukhopadhyay, 1958 ; Maya JASANOFF, Aux marges de l'Empire : conquérants et collectionneurs à l'assaut de l'Orient de 1750-1850, Paris, Editions Heloise d'Ormesson, 2009 (2007). Sur l'envoi de mémoires au ministre de la Marine, voir par exemple Sylvia MURR, L'Inde philosophique entre Bossuet et Voltaire, vol. II, L'indologie du Père Coeurdoux. Stratégies, apologétique et scientificité, Paris, Ecole Française d'Extrême Orient, 1987.

18. Neveu d'Ananda Ranga Pillai.

19. Second du Gouverneur de Pondichéry.

20. Chef d'un Etat féodal subordonné, donc receveur des revenus des territoires. Nallatambi perd certainement le bénéfice de sa charge avec la perte des territoires concernés.

21. Ananda RANGAPILLAI, The Diary (1735-1760), Madras, Superintendant Government Press, vol. X, 1961, p. 7-8.

22. S. Jeyaseela STEPHEN (dir.), The Diary of Rangappa, op. cit., p. 188.

23. Dès le début du siècle par la littérature anglaise. Pour la France, voir Marie FOURCADE, Ines zUPANov (dir.), L'Inde des Lumières. Discours, histoire, savoirs (XVII ${ }^{e}-X I X^{e}$ siècles), Paris, Purusartha, 2013.

24. Thomas METCALF, Ideologies of the Raj, Cambridge, Cambridge University Press, 1997.

25. S. Jeyaseela STEPHEN (dir.), The Diary of Rangappa, op. cit., p. 277.

26. Ananda RANGAPILLAI, Diary, op. cit., IX, p. 111. Les références sont nombreuses dans le journal, voir Julie MARQUET, Les courtiers de la Compagnie française des Indes à Pondichéry (début XVIII - début XIX $X^{e}$ siècle), mémoire de Master 2 Histoire et civilisations comparées, sous la direction de MarieNoëlle BoURGUET, Université Paris Diderot Paris 7, 2011, dactylographié, 223 p. (non publié).

27. Lettres patentes en date du 10 décembre 1751 et arrêt du Conseil d'Etat du Roi du 6 juin 1750, dans Edmond GAUDART, Catalogue des manuscrits des anciennes archives, de l'Inde française, Paris Pondichéry, Leroux - Bibliothèque coloniale, 1922-1942, tome 1, p. 11, n 36. Sur Dupleix, voir l'ouvrage ancien d'Alfred MARTINEAU, Dupleix et l'Inde Française, 1722-1741, Paris, Société d'Editions géographiques, maritimes et coloniales, 1928.

28. Les délibérations du Conseil Supérieur de Pondichéry rendent compte d'un nouvel effort législatif et réglementaire entre 1763 et 1778.

29. ANOM FM SG INDE 379, 420; ANOM Col E 379 Bis; ANOM C2 99, 100, 143.

30. Edmond GAUDART (dir.), Correspondance des agents à Pondichéry de la nouvelle Compagnie des Indes avec les administrateurs à Paris, 1788- 1803, Pondichéry - Paris, Bibliothèque coloniale Leroux, p. 434-501.

31. Susan NEILD-BASU, « The Dubashes of Madras ", Modern Asian Studies, vol. 18, n 1, 1984, p. 17.

32. Simon SCHAFFER et al. (dir.), The Brokered World, op. cit., p. ix.

33. A. KRISHNASAMY PILLAI, «The family of Ananda RangaPillai », art.cit.

34. L'appréciation de l'utilité d'une action et l'ajustement des services rendus aux attentes de bénéfices en retour apparait dans l'analyse de la position des dubashes de Madras de Kanakalatha MUKUND. Elle estime que les dubashes acceptent de prendre à perte les fermes de la Compagnie, de manière à se rendre utiles aux Britanniques, et d'obtenir en contrepartie des privilèges fiscaux ou honorifiques, voir Kanakalatha MUKUND, The Trading World of the Tamil Merchant: Evolution of Merchant Capitalism in the Coromandel, London - Hyderabad, Sangam - Orient Longman, 1999 p. 158-160. La proposition est convaincante, mais la documentation ne permet pas de déterminer si les fermes sont prises à perte à Pondichéry (certaines semblent même constituer une niche lucrative, comme les fermes du tabac, du bétel et des boissons). 
35. INAL 21, 25.

36. Sur les courriers envoyés à Paris ou à Lorient lors de l'affaire Nanyappa, voir Paul OLAGNIER, Les Jésuites à Pondichéry et l'affaire Naniapa, Paris, Leroux, 1932 ; Danna AGMON, An Uneasy Alliance: Traders, Missionaries and Tamil Intermediaries in Eighteenth Century French India, Thèse de Doctorat de l'Université de Michigan, 2011, 317 p. (non publié); sur les certificats, voir INAL 21; GobalakichenANe, La Révolution française des Tamouls de Pondichéry, 1790-1793, mémoire de DEA, Université de Nantes, 1997, 196 p. (non publié), p.146-154. Sur le passage des rapports interpersonnels et des réseaux d'interconnaissance, comme forme de garantie, à l'autorité du document écrit, gardant la mémoire et la trace des positions de chacun, voir Bhavani RAMAN, «Tamil Munshis and Kacceri Tamil under the Company's Document Raj in Early-NineteenthCentury Madras", dans Thomas R. TRAUTMAnN, The Madras School of Orientalism. Producing Knowledge in Colonial South India, Delhi, Oxford University Press, 2009, p. 209-233.

37. INAL 21, 20 ; ANOM Col E 379 Bis.

38. INAL 21, 20.

39. Kapil RAJ, « Mapping Knowledge Go-Betweens in Calcutta, 1770-1820 », dans Simon SCHAFFER et al. (dir.), The Brokered World, op. cit. L'inconsistance des relations et la difficulté du maintien des alliances et de la confiance est caractéristique dans les rapports des Français avec Tipu Sultan, voir par exemple Lettres et conventions des gouverneurs de Pondichéry avec différents princes hindous, 1666-1793, Pondichéry, Société d'histoire de l'Inde française, 1911-1914.

40. Simon SCHAFFER et al. (dir.), The Brokered World, op. cit.

41. Ainsi que Sanjay SUBRAHMANYAm invite à le faire, dans Sanjay SUBRAHMANYAM, « Between a Rock and a Hard Place ", art. cit., p. 438.

42. Pillai évoque l'intérêt de Dupleix pour son journal : « comme mon journal [est] en français, il aimerait le voir ", dans Ananda RANGAPILLAI, The Diary, op. cit.,VIII.

43. Kanakalatha MUKUND, The Trading World of the Tamil Merchant, op. cit., p. 149.

44. Le critère de définition des identités dans les documents produits par ou pour l'administration coloniale semble plutôt être celui de l'étendue des réseaux, de la richesse (Ananda Ranga Pillai, condamnant le « caractère dépravé » de Balu Chetti, un de ses concurrents, affirme que « la nature vile de son caractère est à imputer au fait qu'il n'est pas né riche ", dans Ananda RANGAPILLAI, The Diary, op.cit., I, p.58), ou de la supériorité de la médiation (Tiruvengadam III insiste sur l'ancienneté du poste de courtier en chef dans sa famille, alors que Candappa et son frère ne seraient que des « serviteurs » du gouverneur Law, ceux qui «lavaient ses plats ", dans S. Jeyaseela STEPHEN (dir.), The Diary of Rangappa, op. cit., p. 201-203). Le fait que la caste Idayiar apparait haut-placée dans les classements réalisés à Pondichéry au XIX ${ }^{\mathrm{e}}$ siècle pourrait montrer que la stratégie des dubashes a été payante. Mukund estime que c'est le prestige de la famille Pillai, acquis dans les interactions avec les Français, qui a entraîné cette revalorisation.

45. Kanakalatha MUKUND, The View From Below, op. cit., p. 158-168; David SHULMAN, "Cowherd or King? The Sanskrit Biography of Ananda Ranga Pillai ", dans David ARNOLD, Stuart BLACKBURN (dir.), Telling Lives in India. Biography, Autobiography, and Life History, Bloominton and Indianapolis, Indiana University Press, Permanent Black, 2004, p. 175-203. L'éditeur du Campu mentionne plusieurs poètes patronnés par Pillai, comme Kasturi Rangayya, originaire de Trichinopoly, qui a écrit un traité en télugu sur les mesures poétiques, intitulé Anandarangarat Chadamu, dédié à son mécène, et joué par des musiciens et des danseuses. Il cite également des poètes tamouls ayant bénéficié de la protection du courtier, en particulier Sadasiva Desikar, auteur d'un poème intitulé Anandaranga-k-kovai en son honneur; voir V. RAGHAVAN (dir.), Anandaranga Campu, TrichyMadras, Palaniappa Brothers, 1948, p. 9. 
46. R. SAThyanarayanan, Anandarangavijaya Campu. A Study and Translation, thèse de Philosophie Sanskrite sous la direction de V. K. Sastri, Université de Pondichéry, Département de Sanscrit, 2003 (non publiée).

47. Kanakalatha Mukund souligne l'alliance d'intérêts entre la classe des nouvelles élites et l'Etat colonial au XVIII ${ }^{\mathrm{e}}$ siècle. A Madras comme à Pondichéry, les Européens ont établi des gouvernements dont la légitimité n'est jamais sérieusement remise en question par les populations locales. Pour l'historienne, cela s'explique en partie par leur respect et leur prise en charge du système local complexe, liant relations politiques et sociales. Il s'agirait d'un des traits qui distingue cet Etat colonial de celui, plus mature et assuré, qui s'établit au XIX ${ }^{\mathrm{e}}$ siècle : «les administrateurs européens ont cherché à valider leur autorité auprès de la société indigène en fonctionnant à l'intérieur du système de valeurs de cette société, et en suivant ses traditions ", dans Kanakalatha Mukund, The View From Below, op. cit., p. 170-171.

48. Sanjay Subrahmanyam, "Between a Rock and a Hard Place », art. cit, p. 439. Les deux autres aspects de son expertise, déjà évoqués, sont sa perspicacité financière et banquière et sa capacité à maîtriser les informations stratégiques.

49. Ananda RANGAPILLAI, The Diary, op.cit., VII, p. 382.

50. Règlement de justice en date du 28 janvier 1778, dans Gnanou DiAgou (dir.), Arrêts du Conseil Supérieur de Pondichéry, Pondichéry, Bibliothèque Publique, 1935, tome 3, n 574, p. 380.

51. Pour la même période, voir en particulier les travaux de Catarina Madeira Santos sur l'Angola.

52. Bernard S. COHN, Colonialism and its Forms of Knowledge: the British in India, Princeton, Princeton University Press, 1996. La logique française de respect des us et coutumes pourrait avoir également été progressivement renforcée, après la guerre de sept Ans, par ce que Kate Marsh appelle « le trope de l'Inde perdue » : conscients de la perte de leur empire des Indes, les Français chercheraient à se présenter comme des colonisateurs "justes", et aimés des Indiens, par opposition aux Britanniques qui se seraient devenus les nouveaux despotes de l'Inde, dans Kate MARSH, India in the French Imagination. Peripheral Voices, 1754-1815, Londres, Pickering \& Chatto, 2009. Ce trope est particulièrement pertinent pour analyser les discours et les postures après 1816.

53. Les administrateurs du comptoir doivent revenir sur certaines des limitations qu'ils ont essayé d'imposer au culte hindou, suite à la désertion des corps de métier de la ville, voir les Procès-verbaux des délibérations du Conseil Souverain de la Compagnie des Indes, Pondichéry, Société de l'Histoire de l'Inde Française, 1911-1914, tomes 1 et 2. La menace de désertion persiste jusqu'au XIX ${ }^{\mathrm{e}}$ siècle.

54. Sur la différence de positionnement des autorités britanniques, voir notamment Christopher A. BAYLY, Empire and Information. Intelligence gathering and social communication in India, 1780-1870, New Delhi, Cambridge University Press, 2007 (1999); Thomas R. TRAUTMAnN, The Madras School of Orientalism, op. cit. Sur l'élaboration des savoirs orientalistes dans l'empire français au XVIII siècle, voir les travaux fondateurs de Sylvia Murr, et, pour le XIX siècle, Roland LARDINoIS, L'invention de l'Inde. Entre ésotérisme et science, Paris, CNRS Éditions, 2007.

55. Le Conseil supérieur statue également pour certains cas en appel. Le tribunal de la Chaudrie est une institution ancienne, maintenue tout au long du XVIII ${ }^{e}$ siècle par le gouvernement français. Une partie des jugements de ce tribunal a été éditée, voir Jean-Claude BonNAN, Jugements du Tribunal de la Chaudrie de Pondichéry, 1766-1817, Pondichéry, Institut Français de Pondichéry, Ecole Française d'Extrême-Orient, Imprimerie de Sri Aurobindo Ashram, 1999. Il est très probable que l'essentiel des conflits n'était pas porté devant la justice coloniale.

56. Ou « chef des Noirs » ou « chef des Indiens ». 
57. Ananda RANGAPILlAI, The Diary, op.cit., IX, p. 133-134. Il dit également recevoir l'aval de plusieurs gouverneurs pour organiser la censure, et emprisonner de manière arbitraire, autant de prérogatives de l'autorité supérieure, dans Ananda RANGAPILLAI, The Diary, op.cit., X, p. 46.

58. Pondichéry compterait environ 100000 habitants vers 1740 , dont 1500 à 2000 Français, dans Claude MARKovits, Histoire de l'Inde Moderne, 1480-1950, Paris, Fayard, 1994, p. 254. A Madras, si les courtiers de la Compagnie ou des gouverneurs tenaient officieusement le rôle de gouverneurs des Indiens, ce rôle est toujours resté informel, voir NEILD-BASU, « The Dubashes », art.cit., p. 22.

59. INAL 21, 25.

60. INAL 21, 25.

61. Selon l'expression de Nicolas Dirks, dans NIELS BRIMNES, Constructing the Colonial Encounter: Right and Left Hand Castes in Early Colonial South India, Nordic Institute of Asian Studies, Monograh $\mathrm{n}^{\circ} 81$, Richmond, Curzon, 1999.

62. INAL 21, 112 ; INAL 21, 526 ; INAL 21, 543.

63. Georg SIMMEL, Sociologie, op.cit.; Michel FORSE, «Les réseaux sociaux chez Simmel: les fondements d'un modèle individualiste et structural ", dans Lilyane DEROCHE-GURCEL et Patrick WATIER (dir.), La Sociologie de Georg Simmel, Paris, PUF, 2002.

64. « Enregistrement provisoire de Police », dans Gnanou DIAGOU (dir.), Arrêts du Conseil Supérieur, op. cit., tome $3, \mathrm{n}^{\circ} 678$, p. 449.

65. Cette logique est manifeste dans l'organisation de l'espace: la documentation distingue en effet la «ville blanche », habitée par les Français, de la "ville noire », habitée par les Indiens, séparées par un canal. Au milieu du siècle, le Conseil supérieur cherche à interdire aux Indiens d'habiter ou de posséder des terrains dans la "ville blanche ", mais le papier-terrier de 1777 montre que les résistances ont été nombreuses, dans Jacques WEBER, Les relations entre l'Inde et la France, op.cit., p. 64 ; Jean DELOCHE, Le papier terrier de la ville blanche de Pondichéry, 1777, Pondichéry, Institut Français de Pondichéry, 2002.

66. Adrian CARTON, «Shades of Fraternity: Creolization and the Making of Citizenship in French India, 1790-1792 », French Historical Studies, vol. 31, n 4, 2004.

67. Jane BURBANK, Frederick COOPER, Empires, op. cit.

\section{RÉSUMÉS}

Les dubashes, intermédiaires indiens au service de l'administration française, jouent un rôle clé à Pondichéry dans la seconde moitié du XVIII ${ }^{\mathrm{e}}$ siècle. Ce rôle évolue au cours de la période, marquée par les conflits avec l'Angleterre et les difficultés économiques du comptoir. L'article envisage la manière dont la position des dubashes est construite à travers les interactions avec l'administration coloniale. La position des agents indiens est instable en raison de fortes concurrences et de la défiance du gouvernement français. Les dubashes de la famille de Tiruvengadam cherchent à limiter l'instabilité à laquelle ils sont confrontés par des stratégies liées à l'écrit. Ils restent des agents indispensables aux Français. Leur connaissance des structures sociales locales et des formes culturelles est en effet nécessaire à l'exercice de l'autorité.

The dubashes, indian intermediaries serving the French administration, played a key role in Pondicherry during the second half of the eighteenth century, a role that evolved throughout this period marked by conflicts with the British and economic difficulties in the colony. This 
paper examines the construction of the dubashes' position through interactions with the colonial administration. Intense competition among Indian agents, as well as distrust on the part of the French governement, made this role an unstable one. The dubashes of Tiruvengadam's family sought to limit the instability they confronted through the use of the strategic use of the written word. They remained essential agents for the French, as their knowledge of local social structures and cultural forms was necessary for the exercize of the colonial authority.

\section{INDEX}

Mots-clés : Dubashes, intermédiaires, administration coloniale, interactions, processus de délégation, autorité coloniale

Keywords : Dubashes, go-betweens, colonial administration, interactions, delegation process, colonial authority

\section{AUTEUR}

\section{JULIE MARQUET}

Doctorante, Laboratoire ICT

Université Paris Diderot - Paris 7 